\title{
A Dialog Control Algorithm and Its Performance
}

\author{
Ronnie W. Smith* \\ Dept. of Computer Science \\ Duke Universtiy \\ Durham, NC 27706 \\ rws@cs.duke.edu
}

\author{
D. Richard Hipp \\ Dept. of Computer Science \\ Duke Universtiy \\ Durham, NC 27706 \\ drh@cs.duke.edu
}

\author{
Alan W. Biermann \\ Dept. of Computer Science \\ Duke Universtiy \\ Durham, NC 27706 \\ awb@cs.duke.edu
}

\begin{abstract}
A pragmatic architecture for voice dialog machines aimed at the equipment repair problem has been implemented which exhibits a number of behaviors required for efficient humanmachine dialog. These behaviors include:

(1) problem solving to achieve a target goal,

(2) the ability to carry out subdialogs to achieve appropriate subgoals and to pass control arbitrarily from one subdialog to another,

(3) the use of a user model to enable useful verbal exchanges and to inhibit unnecessary ones,

(4) the ability to change initiative from strongly computer controlled to strongly user controlled or somewhere in between, and

(5) the ability to use context dependent expectations to correct speech recognition and track user movement to new subdialogs.
\end{abstract}

A description of the implemented dialog control algorithm is given; an example shows the fundamental mechanisms for achieving the listed behaviors. The system implementation is described, and results from its performance in 141 problem solving sessions are given.

\section{A Voice-Interactive Dialog Machine}

A limited vocabulary voice dialog system designed to aid a user in the repair of electronic circuits has been constructed in our laboratory. The system contains a model of the device to be repaired, debugging and repair procedures, voice recognition and sentence processing mechanisms, a user model, language generation capabilities, and a dialog control system which orchestrates the behaviors of the various parts. This paper describes the

*This research was supported by National Science Foundation Grant Number NSF-IRI-88-03802 and by Duke University. dialog control algorithm and the performance of the total system in aiding a series of subjects in the repair of an electronic circuit.

\section{Target Behaviors}

The purpose of the dialog control algorithm is to direct the activities of the many parts of the system to obtain efficient human-machine dialog. Specifically, it is aimed at achieving the following behaviors.

Convergence to a goal. Efficient dialog requires that each participant understand the purpose of the interaction and have the necessary prerequisites to cooperate in its achievement. This is the intentional structure of [Grosz and Sidner, 1986], the goal-oriented mechanism that gives direction to the interaction. The primary required facilities are a problem solver that can deduce the necessary action sequences and a set of subsystems capable of carrying out those sequences.

Subdialogs and effective movement between them. Efficient human dialog is usually segmented into utterance sequences, subdialogs, that are individually aimed at achieving relevant subgoals ([Grosz, 1978], [Linde and Goguen, 1978], [Polanyi and Scha, 1983], and [Reichman, 1985]). These are called "segments" by [Grosz and Sidner, 1986] and constitute the linguistic structure defined in their paper. The global goal is approached by a series of attempts at subgoals each of which involves a set of interactions, the subdialogs.

An aggressive strategy for global success is to choose the most likely subgoals needed for success and carry out their associated subdialogs. As the system proceeds on a given subdialog, it should always be ready to abruptly drop it if some other subdialog suddenly seems more appropriate. This leads to the fragmented style that so commonly appears in efficient human communication. A subdialog is opened which leads to another, then another, then a jump to a previously opened subdialog, and so forth, in an unpredictable order until the necessary subgoals have been solved for an overall success.

Accounting for user knowledge and abilities. Cooperative problem solving involves maintaining a dynamic profile of user knowledge, termed a user model. This concept is described for example in [Kobsa and Wahlster, 1988] and [Kobsa and Wahlster, 1989], [Chin, 1989], [Cohen and Jones, 1989], [Finin, 1989], [Lehman and Carbonell, 1989], [Morik, 1989], and [Paris, 1988]. The user 
model specifies information needed for efficient interaction with the conversational partner. Its purpose is to indicate what needs to be said to the user to enable the user to function effectively. It also indicates what should be omitted because of existing user knowledge.

Because considerable information is exchanged during the dialog, the user model changes continuously. Mentioned facts are stored in the model as known to the user and are not repeated. Previously unmentioned information may be assumed to be unknown and may be explained as needed. Questions from the user may indicate lack of knowledge and result in the removal of items from the user model.

Change of initiative. A real possibility in a cooperative interaction is that the user's problem solving ability, either on a given subgoal or on the global task, may exceed that of the machine. When this occurs, an efficient interaction requires that the machine yield control so that the more competent partner can lead the way to the fastest possible solution. Thus, the machine must be able to carry out its own problem solving process and direct the actions to task completion or yield to the user's control and respond cooperatively to his or her requests. This is a mixed-initiative dialog as studied by [Kitano and Van Ess-Dykema, 1991], [Novick, 1988], [Whittaker and Stenton, 1988], and [Walker and Whittaker, 1990]. As a pragmatic issue, we have found that at least four initiative modes are useful:

(1) directive - The computer has complete dialog control. It recommends a subgoal for completion and will use whatever dialog is necessary to obtain the needed item of knowledge related to the subgoal.

(2) suggestive - The computer still has dialog control, but not as strongly. The computer will make suggestions about the subgoal to perform next, but it is also willing to change the direction of the dialog according to stated user preferences.

(3) declarative - The user has dialog control, but the computer is free to mention relevant, though not required, facts as a response to the user's statements.

(4) passive - The user has complete dialog control. The computer responds directly to user questions and passively acknowledges user statements without recommending a subgoal as the next course of action.

Expectation of user input. Since all interactions occur in the context of a current subdialog, the user's input is far more predictable than would be indicated by a general grammar for English. In fact, the current subdialog specifies the focus of the interaction, the set of all objects and actions that are locally appropriate. This is the attentional structure described by [Grosz and Sidner, 1986] and its most important function in our system is to predict the meaning structures the user is likely to communicate in a response. For illustration, the opening of a chassis cover plate will often evoke comments about the objects behind the cover; the measurement of a voltage is likely to include references to a voltmeter, leads, voltage range, and the locations of measurement points.

The subdialog structure thus provides a set of expected utterances at each point in the conversation and these have two important roles:

(1) The expected utterances provide strong guidance for the speech recognition system so that error correction can be maximized. Where ambiguity arises, recognition can be biased in the direction of meaningful statements in the current context. Earlier researchers who have investigated this insight are [Erman et al., 1980], [Walker, 1978], [Fink and Biermann, 1986], [Mudler and Paulus, 1988], [Carbonell and Pierrel, 1988], [Young et al., 1989], and [Young and Proctor, 1989].

(2) The expected utterances from subdialogs other than the current one can be used to indicate that one of those others is being invoked. Thus, expectations are one of the primary mechanisms needed for tracking the conversation as it jumps from subdialog to subdialog. This is known elsewhere as the plan recognition problem and it has received much attention in recent years. See, for example, [Allen, 1983], [Litman and Allen, 1987], [Pollack, 1986], and [Carberry, 1990].

Systems capable of all of the above behaviors are rare as has been observed by [Allen et al., 1989]: "no one knows how to fit all of the pieces together." One of the contributions of the current work is to present an architecture that can provide them all in the limited domain of electric circuit repair. [Allen et al., 1989] describe their own architecture which concentrates on representations for subdialog mechanisms and their interactions with sentence level processing. Our work differs from theirs on many dimensions including our emphasis on achieving mixed-initiative, real time, voice interactive dialog which utilizes a user model.

\section{The Zero Level Model}

The system implemented in our laboratory (described in great detail in [Smith, 1991]) achieves the above behaviors sufficiently to enable efficient human-machine di. alogs in the electric circuit repair environment. The com. plexity of the system prevents its complete descriptior here. However, a zero level model has been devised for pedagogical purposes which illustrates its principles o operation. This model mimicks the mechanism of the dialog machine while omitting the huge array of details necessary to make a real system work. A later sectior in this paper describes the actual implementation anc some of its major modules.

The zero level model is the recursive subroutine Zmod Subdialog shown in figure 1 . This routine is entered witl a single argument, a goal to be proven, and its action: are to carry out a Prolog-style proof of the goal. A sid. effect of the proof may be a resort to voice interaction: with the user to supply necessary missing axioms. II 


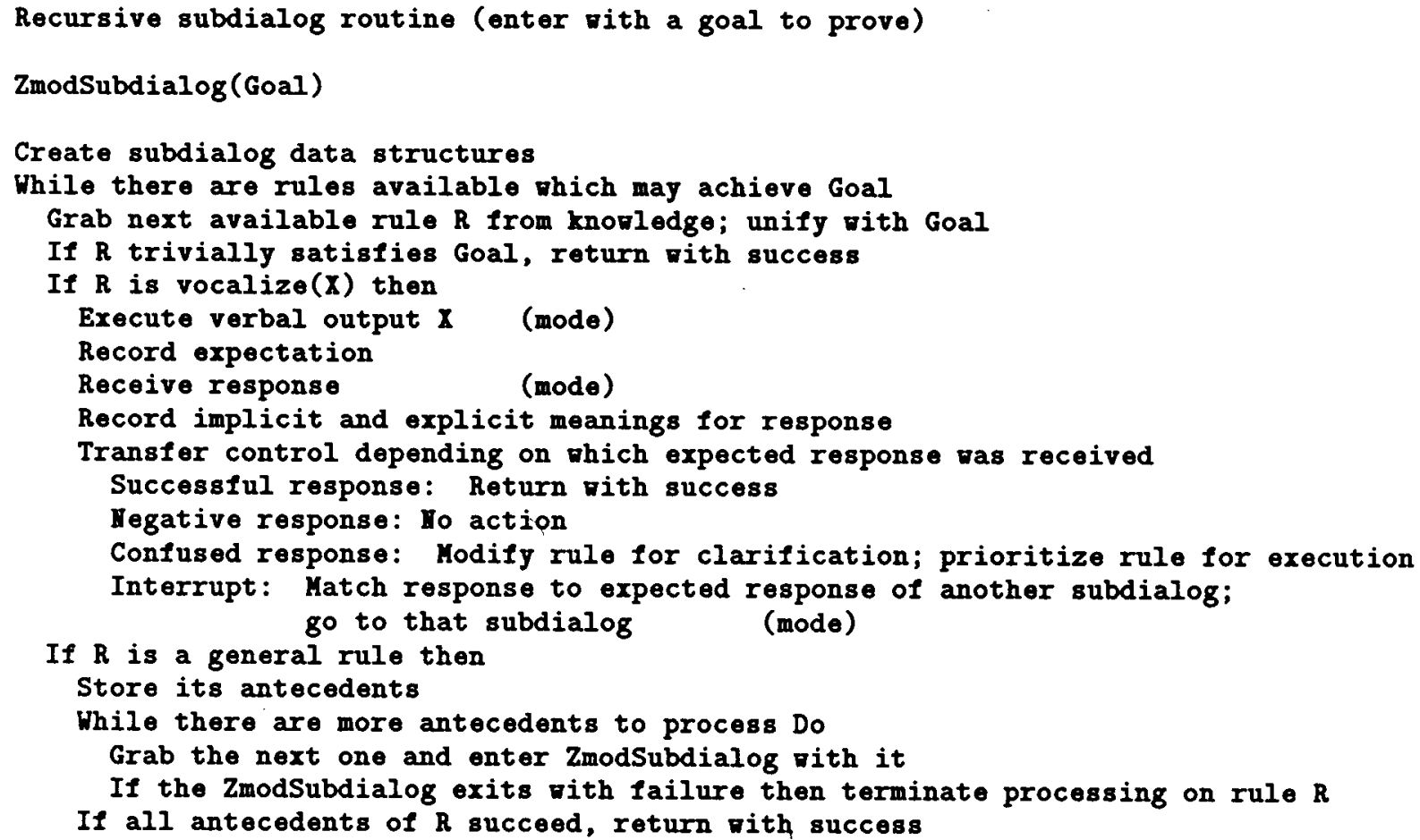

HOTE: SUCCESSFUL COMPLETIOH OF THIS ROUTIYE DOES MOT MECESSARILY MEAM TRAHSFER OF CONTROL TO THE CALLIMg ROUTINE. COHTROL PASSES TO THE SUBdIALOGUE SELECTED BY THE DIALOG COMTROLLER.

Figure 1: Zero Level Model

fact, the only voice interactions the system undertakes are those called for by the theorem proving machinery.

The ZmodSubdialog routine has a unique capability needed for proper modeling of subdialog behaviors. Specifically, its actions may be suspended at any time so that control may be passed to another subdialog, another instantiation of $\mathrm{ZmodSubdialog}$ that is aimed at achieving another goal. However, control may at a later time return to the current instantiation to continue its execution. In fact, a typical computation involves a set of ZmodSubdialog instantiations all advanced to some point in the proofs of their respective goals; control passes back and forth between them looking for the most likely chances of success until, finally, enough goals are proven to complete the global proof.

The algorithm becomes understandable if an example is followed through in full detail. Assume that the following database of Prolog-like rules are contained in the system knowledge base.

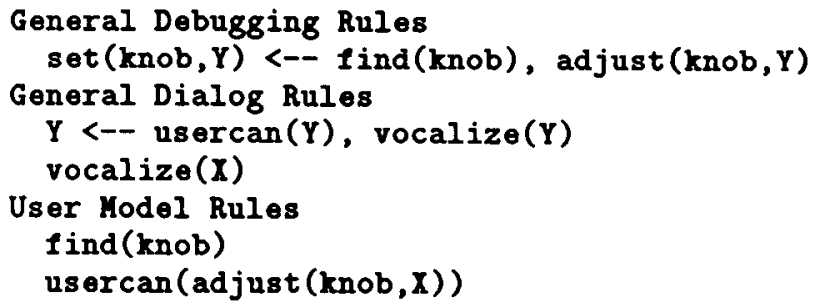

Further assume that $\mathrm{ZmodSubdialog}$ is entered with argument Goal $=\operatorname{set}(\mathbf{k n o b}, 10)$. Then in Prolog fashion, the routine grabs the rule $R$ : set(knob, $Y)$ - find(knob), adjust(knob, $Y)$, and attempts to prove set(knob,10). The algorithm offers three choices depending on whether $R$ is trivial (a single predicate which is not vocalize $(X)$ ), $R$ is vocalize $(X)$, or $R$ is a rule with antecedents as is the case here. Thus, in this case the third alternative is followed, and the two antecedents are queued for sequential execution (find(knob) and adjust(knob,10)). Then the first antecedent is selected and ZmodSubdialog is entered with argument Goal $=$ find $(k n o b)$.

The new subdialog to achieve find(knob) is short, however, since the user model indicates the user already knows how to find the knob because find(knob) exists as an available rule. In fact, $\mathrm{ZmodSubdialog}$ finds find(knob) in the rule base, enters the first choice (trivial) and returns with success. If find(knob) had not been found in the user model, the system might have engaged in dialog to achieve this goal. The subdialog control mechanism is not obligated to pass control back to the calling routine, but in this example we will assume that it does.

Satisfactory proof of find $(\mathrm{knob})$ means that the next antecedent at this level, adjust(knob,10), can be attempted, and ZmodSubdialog is entered with this goal. Here, our model selects $R=Y \leftarrow \operatorname{usercan}(Y)$, vocalize( $Y$ ), unifying $Y$ with adjust $(k n o b, 10)$. Then a deeper call to $\mathrm{ZmodSubdialog}$ finds usercan(adjust $(\mathrm{knob}, \mathrm{X})$ ) in 
the user model which means control passes to the antecedent vocalize(adjust(knob,10)). This yields another entry to ZmodSubdialog and a selection of the second branch. Here the system executes a voice output to satisfy vocalize(adjust(knob,10)): "Set the knob to one zero."

The handling of the goal find(knob) illustrates how the user model can act to satisfy the theorem proving process and prevent the enunciation of unneeded information. As the theorem proving process proceeds, the fact that a user knows something is represented in the knowledge base as an achieved goal. Theorem proving will encounter this and proceed without voice interaction. In the example, the model already indicates that the user knows how to find the knob so no voice interaction is needed for this goal.

The handling of the goal adjust(knob,10) illustrates how the user model supports the theorem proving process by enabling voice dialog when it is needed. This goal could not be proven by application of rules available in the database and the proof was blocked from further progress. In our terminology, there was a "missing axiom" for the proof. So the system must either give up on this proof or try to fill in the missing axiom by resorting to voice dialog. In the current case, voice dialog was enabled by the user model fact usercan(adjust(knob,X)). This fact opens the way for a query to the user. If the query is positively answered, then the missing axiom is made available.

The role of the user model is thus to supply or fail to supply axioms at the bottom of the proof tree. It both inhibits extraneous verbalism and enables interactions necessary to prove the theorem.

Returning to the example computation of ZmodSubdialog, a voice output has just been given, and the system then records, for this output, the set of expected responses:

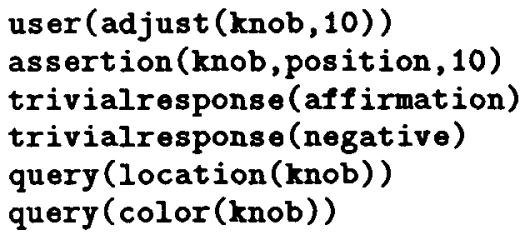

Expected responses are compiled from the domain knowledge base and from the dialog controller knowledge base.

The user's response is then matched (unified) against the expected meanings and subsequent actions depend on which meaning fits best. Four different types of actions may occur at this point.

(1) The user might respond with some paraphrase of "I set the knob to one zero," or "Okay", which would be interpreted as successful responses. The routine ZmodSubdialog would return with success.

(2) The user might also answer "No" yielding a failure and another cycle around the theorem proving loop looking for an applicable rule.

(3) The user might respond with "What color is the knob?" indicating there may be a chance for a success here if there is further dialog. In fact, our system handles such a need for clarification by dynamically modifying the rule and reexecuting with a newly required clarification subdialog. Here the rule set $(k n o b, 10)$ $\leftarrow$ find(knob), adjust(knob,10) becomes modified to $\operatorname{set}(k n o b, 10) \leftarrow$ find $(k n o b)$, vocalize(knob, white), adjust(knob,10). Reexecution will then yield an explanation of the knob color followed by the previous request reenunciated: "Set the knob to one zero."

(4) The user might respond with an utterance that matches no local expectation. Here the system examines expectations of other subdialogs and searches for an acceptable match. If one is found, control will pass to that subdialog. For example, if the response is, "The LED is flashing seven," and if this is an appropriate response in some other subdialog, control will pass to it.

The control of initiative in ZmodSubdialog is handled by special processing at the steps marked "mode." Thus, in strongly directive mode, verbal outputs will be very positively stated, responses will be expected to be obedient, and interrupts to other subdialogs will be restricted. In less demanding modes, outputs will be weaker or merely suggestive, a wider range of responses will be allowed, and transitions to other subdialogs will be more freely permitted. In the most passive mode, there are few outputs except answers to questions, and an interrupt to any subdialog is acceptable.

A very important part of the dialog system is the domain processor, the application dependent portion of the system. It receives all information related to the current problem and suggests debugging steps to carry the process further. We model calls to this processor with the rule: debug $(X) \leftarrow$ (debuggingstep $(X))^{*}$. This rule is called upon to debug device $X$ with the predicate $\operatorname{debug}(X)$ and its effect is to specify a sequence of debugging steps which will be specified dynamically as the problem unfolds and which will lead to repair of the device.

\section{The Implementation}

\subsection{An Integrated Architecture}

The implemented system is based on a computational model for dialog processing presented in [Smith, 1991]. The model is applicable to the general class of taskoriented dialogs, dialogs where the computer is assisting the user in completing a task as the dialog ensues. The derived implementation consists of the following modules:

dialog controller - This is the supervisor of the dialog processing system. It provides the subdialog processing highlighted in the zero level model. In addition, it provides the complex algorithm required to properly handle arbitrary clarification subdialogs and interrupts as well as provide the dialog expectations needed to 
assist with input interpretation. It also maintains all dialog information shared by the other modules and controls their activation.

domain processor - As previously mentioned, it provides suggestions about debugging steps to pursue. In our system the domain processor assists users in circuit repair. It receives user-supplied domain information from the dialog controller and returns suggested debugging subgoals to the controller for consideration. The subgoal selection process weighs the level of expected usefulness of a subgoal with the number of times the subgoal has been previously selected. Consequently, the module may recommend challenging previously reported information if no noticeable progress in the task is being made. In this manner, the module and system can recover from erroneous inputs. If the dialog system is to be used to repair other devices, this is the module that needs to be replaced.

knowledge - This is the repository of information about task-oriented dialogs including: (1) rules for proving completion of goals; (2) user model information including a set of rules for inferring user model information from user input; (3) rules for determining when a clarification subdialog should be initiated; and (4) rules for defining the expectations for the user's response as a function of the type of goal being attempted. Note that the predefined information of this module is easily modified without requiring changes to the dialog controller.

theorem prover - This provides the general reasoning capability of the system. In order to allow the dialog controller to control the potentially arbitrary movement among subdialogs, the theorem prover has been made interruptible and put under the supervision of the dialog controller. Consequently, the theorem prover can maintain a set of partially completed proofs, and can activate different proofs as instructed by the dialog controller. It can also dynamically modify the proof structure, a key feature for handling clarification subdialogs. Foremost, the theorem prover is able to suspend itself when it encounters a missing axiom, permitting natural language interaction to assist in axiom acquisition. This contrasts with traditional theorem proving approaches (e.g. Prolog) which simply engage in backtracking when a missing axiom is encountered.

linguistic interface - This consists of the generation and recognition modules. They use information on context and expectation as provided by the dialog controller. The linguistic generator was developed by Robin Gambill. It uses a rule-driven approach that takes as input an utterance specification which encapsulates the desired meaning and produces as output an
English string that is sent to a text-to-speech converter for enunciation. Various approaches to generation have been described in [Danlos, 1987], [Hovy, 1988], [Jacobs, 1987], [McKeown, 1985], and [Patten, 1988]. The recognition module was designed to be able to recover from ungrammatical inputs. It will be described in greater detail below.

\subsection{Minimum distance parsing}

A challenging design problem in any natural language system is the development of a parser which will translate the lattice of words output by the speech recognizer into a phrase of some synthetic language (Prolog in our instance) which encapsulates the meaning of what was originally spoken. The design difficulty is exacerbated by the fact that due to speech recognition errors, the word lattice output by the speech recognizer is probably different from what the user spoke, and the fact that users will sometimes speak with a word order or sentence construction which the designers of the parser's grammar did not forsee. (These, and other problems associated with robust parsing of speech are further described in [Eastman and McLean, 1981], [Hayes et al., 1986], and [Young et al., 1989].) In our system, parsing is accomplished using a minimum-distance parsing algorithm, similar to algorithms described in [Aho and Peterson, 1972], [Lyon, 1974], and [Levinson, 1985], but extended to accept a lattice as input, instead of a simple word list, and also extended to simultaneously perform syntax-directed translation [Aho and Ullman, 1969] into the target language. Minimum-distance parsing finds strings in a given context-free language which can be converted to a string of the input lattice with a minimum number of weighted insertions and deletions. When two or more strings are equidistant from the input, dialog expectation is used to break the tie. The minimumdistance parsing algorithm is very robust - it always finds at least one parse - but it is also computationally expensive. Our algorithm is faster than previously reported parsing algorithms of the same generality, but it still required careful hand-crafting and optimization on a fast machine in order to provide real-time response. It should also be noted that our recognition system is capable of selective verification of meaning. This is highlighted in the following sample dialogs.

\section{Sample Dialogs}

Figure 2 shows two dialogs obtained from actual usage of the system. These dialogs can be seen in [Hipp and Smith, 1991] that is part of the video presentation of the conference. The words in parentheses found beside certain user statements are the words actually provided by the speech recognizer. Where the discrepancy between the output of the speech recognizer and the words produced by the user is insignificant, they have been omitted.

The first dialog is an entire dialog conducted in declarative mode. Particular points to notice from this usercontrolled dialog are: 
Dialog 1 - Declarative Mode (C denotes the computer, U the user, and $R$ the recognizer)

1. C: This is the circuit $f i x$ it shop. Hor may I help you?

2. U: I vant to fix this rs111.

3. C: I am familiar with that circuit. The LED is supposed to be displaying alternately flashing one and seven.

4. U: The LED is displaying an alternately flashing one and the top corner of a seven.

5. C: There is supposed to be a vire between connector nine nine and connector one zero zero.

6. U: It is there.

7. C: There is supposed to be a wire between connector nine nine and connector one zero two.

8. U: There is no wire on connector one zero four. (R: stays know vire i connector one zero for)

9. C: There is supposed to be a vire between connector one zero four and connector one zero two. [User adds wire and verifies the circuit is repaired, a three step process.]

10. $U$ : The circuit is norking.

11. C: Glad to have been of assistance. Good-bye.

Dialog 2 - Directive Mode excerpt (begins just after computer describes what circuit does)

8. U: The LED is displaying nothing.

9. C: What is the siitch at when the LED is off?

10: U: The switch is down.

11. C: Put the switch up.

12. U: Done.

13. C: Put the knob to one zero.

14. U: Which knob?

15. C: Near the top boundary and in the center is a green region. Nor, in the middle right of this green region is the control knob.

16. U: The knob is at one zero.

17. C: What is the LED displaying?

18. U: The LED is displaying a one and seven at the same time.

19. C: Put the knob to zero.

20. U: Done.

21. C: What is the LED displaying?

22. U: The same thing. ( $R$ : the same same)

23. C: Did you mean to say the LED is displaying the same thing?

24. U: Yes.

[Eventually, a missing vire is detected and added, and the computer explicitly guides the user through the verification process. The entire dialog lasts 51 utterances.]

Figure 2: Sample Dialogs

(1) Successful processing of anaphoric reference at utterance 6 .

(2) Successful shifts to user-initiated subdialogs at utterances 8 and 10.

(3) Successful recovery from speech recognition errors at utterance 8 .

The second dialog is an excerpt from a dialog conducted in directive mode (strongly computer-controlled dialog). The total dialog lasted 51 utterances in contrast to the 11 utterance declarative mode dialog. Particular points to notice from this excerpt include:

(1) Computer responses which are more directed and forceful in content than in dialog 1 .

(2) Successful handling of a clarification subdialog (utterances 13-16).

(3) Successful verification of the implicit meaning of a user utterance in the presence of speech recognition errors in utterance 22 . In contrast with utterance 8 of dialog 1 , the system decided an explicit verification subdialog was required to ascertain the meaning of the user's utterance.

\section{Experimental Results}

The system has been implemented on a Sun 4 workstation with the majority of the code written in Quintus Prolog ${ }^{1}$, and the parser in C. Speech recognition is performed by a Verbex 6000 running on an IBM PC and speech production is performed by a DECtalk ${ }^{2}$ DTCO1 text-to-speech converter. The users are restricted to a 125 word vocabulary in the connected speech system.

\footnotetext{
${ }^{1}$ Quintus Prolog is a trademark of Quintus Computer Systems, Incorporated

${ }^{2}$ DECtalk is a trademark of Digital Equipment Corporation.
} 
The implemented domain processor has been loaded with a model for a particular experimental circuit assembled on a Radio Shack 160-in-One Electronic Project Kit.

After testing system prototypes with a few volunteers, eight subjects used the system during the formal experimental phase. After a warmup session where the subject trained on the speech recognizer and practiced using the system, each subject participated in two sessions where up to ten problems were attempted. The system ran in declarative mode (user-controlled dialogs) during one session and in directive mode (strongly computercontrolled dialog) in the other session. Subjects attempted a total of 141 dialogs of which 118 or $84 \%$ were completed successfully. ${ }^{3}$ Subjects spoke a total of 2840 user utterances, with $81.5 \%$ correctly interpreted by the system although only $50.0 \%$ were correctly recognized word for word by the speech recognizer. The average speech rate was 2.8 sentences per minute, and the average task completion times for successful dialogs were 4.5 and 8.5 minutes, respectively, for declarative and directive modes. The average number of user utterances per successful dialog was 10.7 in declarative mode and 27.6 in directive mode. A detailed description of the experiment and results is given in [Smith, 1991]. The substantially shorter completion times for users in declarative mode can be attributed to the fact that the subjects learned many of the debugging procedures during the experiment and did not need the detailed descriptions given in the directive mode.

\section{Summary}

A voice interactive dialog architecture has been developed which achieves simultaneously a variety of behaviors believed to be necessary for efficient human-machine dialog. Goal oriented behavior is supplied by the theorem proving paradigm. Subdialogs and movement between them is implemented with an interruptible theorem prover that maintains a set of partially completed proofs and can work on the most appropriate one at any given time. A user model is provided by a continuously changing set of rules that are referenced in the theorem proving process either to enable or inhibit voice dialog. Mixed initiative is made possible by variable types of processing by the output and input routines and by restricting or releasing the ability to interrupt to a new subdialog. Expectation is associated with individual subdialogs, is compiled from domain and dialog information related to each specific output, and is used to improve voice recognition and enable movement between subdialogs.

\footnotetext{
${ }^{3}$ Of the 23 dialogs which were not completed, 22 were terminated prematurely due to excessive time being spent on the dialog. Misunderstandings due to misrecognition were the cause in 13 of these failures. Misunderstandings due to inadequate grammar coverage occurred in 3 of the failures. In 4 of the failures the subject misconnected a wire. In one failure there was confusion by the subject about when the circuit was working, and in another failure there were problems with the system software. A hardware failure caused termination of the final dialog.
}

\section{References}

[Aho and Peterson, 1972] Alfred V. Aho and Thomas G. Peterson. A minimum distance error-correcting parser for context-free languages. SIAM Journal on Computation, 1(4):305-312, 1972.

[Aho and Ullman, 1969] A. V. Aho and J. D. Ullman. Properties of syntax directed translations. Journal of Computer and System Sciences, 3(3):319-334, 1969.

[Allen et al., 1989] J. Allen, S. Guez, L. Hoebel, E. Hinkelman, K. Jackson, A. Kyburg, and D. Traum. The discourse system project. Technical Report 317, University of Rochester, November 1989.

[Allen, 1983] J.F. Allen. Recognizing intentions from natural language utterances. In M. Brady and R.C. Berwick, editors, Computational Models of Discourse, pages 107-166. MIT Press, Cambridge, Mass., 1983.

[Carberry, 1990] S. Carberry. Plan Recognition in Natural Language Dialogue. MIT Press, Cambridge, Mass., 1990.

[Carbonell and Pierrel, 1988] N. Carbonell and J.M. Pierrel. Task-oriented dialogue processing in humancomputer voice communication. In H. Niemann, M. Lang, and G. Sagerer, editors, Recent Advances in Speech Understanding and Dialog Systems, pages 491-496. Springer-Verlag, New York, 1988.

[Chin, 1989] D.N. Chin. KNOME: Modeling what the user knows in UC. In A. Kobsa and W. Wahlster, editors, User Models in Dialog Systems, pages 74-107. Springer-Verlag, New York, 1989.

[Cohen and Jones, 1989] R. Cohen and M. Jones. Incorporating user models into expert systems for educational diagnosis. In A. Kobsa and W. Wahlster, editors, User Models in Dialog Systems, pages 313333. Springer-Verlag, New York, 1989.

[Danlos, 1987] L. Danlos. The Linguistic Basis of Text Generation. Cambridge University Press, New York, 1987.

[Eastman and McLean, 1981] C. M. Eastman and D. S. McLean. On the need for parsing ill-formed input. American Journal of Computational Linguistics, $7(4): 257,1981$.

[Erman et al., 1980] L.D. Erman, F. Hayes-Roth, V.R. Lesser, and D.R. Reddy. The Hearsay-II speechunderstanding system: Integrating knowledge to resolve uncertainty. ACM Computing Surveys, pages 213-253, June 1980.

[Finin, 1989] T.W. Finin. GUMS: A general user modeling shell. In A. Kobsa and W. Wahlster, editors, User Models in Dialog Systems, pages 411-430. SpringerVerlag, New York, 1989.

[Fink and Biermann, 1986] P.E. Fink and A.W. Biermann. The correction of ill-formed input using history-based expectation with applications to speech understanding. Computational Linguistics, 12(1):13$36,1986$. 
[Grosz and Sidner, 1986] B.J. Grosz and C.L. Sidner. Attentions, intentions, and the structure of discourse. Computational Linguistics, 12(3):175-204, 1986.

[Grosz, 1978] B.J. Grosz. Discourse analysis. In D.E. Walker, editor, Understanding Spoken Language, pages 235-268. North-Holland, New York, 1978.

[Hayes et al., 1986] Philip J. Hayes, Alexander G Hauptmann, Jaime G. Carbonell, and Masaru Tomita. Parsing spoken language: A semantic caseframe approach. In COLING-86: Proceedings of the 11th International Conference on Computational Linguistics, pages 587-592, Bonn, August 1986.

[Hipp and Smith, 1991] D. R. Hipp and R. W. Smith. A demonstration of the "circuit fix-it shoppe". A 12 minute videotape available from the authors at Duke University, Durham, NC 27706, August 1991.

[Hovy, 1988] E.H. Hovy. Generating Natural Language under Pragmatic Constraints. Lawrence Erlbaum Associates, Hillsdale, NJ, 1988.

[Jacobs, 1987] P.S. Jacobs. KING: a knowledgeintensive natural language generator. In Gerard Kempen, editor, Natural Language Generation, pages 219230. Martinus Nijhoff Publishers, Boston, 1987.

[Kitano and Van Ess-Dykema, 1991] H. Kitano and C. Van Ess-Dykema. Toward a plan-based understanding model for mixed-initiative dialogues. In Proceedings of the 29th Annual Meeting of the Association for Computational Linguistics, pages 25-32, 1991.

[Kobsa and Wahlster, 1988] A. Kobsa and W. Wahlster, editors. Special Issue on User Modeling. MIT Press, Cambridge, Mass., September 1988. A special issue of Computational Lingusitics.

[Kobsa and Wahlster, 1989] A. Kobsa and W. Wahlster, editors. User Models in Dialog Systems. SpringerVerlag, New York, 1989.

[Lehman and Carbonell, 1989] J.F. Lehman and J.G. Carbonell. Learning the user's language: A step towards automated creation of user models. In A. Kobsa and W. Wahlster, editors, User Models in Dialog Systems, pages 163-194. Springer-Verlag, New York, 1989.

[Levinson, 1985] Stephen E. Levinson. Structural methods in automatic speech recognition. Proceeding of the IEEE, 73(11):1625-1650, 1985.

[Linde and Goguen, 1978] C. Linde and J. Goguen. Structure of planning discourse. J. Social Biol. Struct., 1:219-251, 1978.

[Litman and Allen, 1987] D.J. Litman and J.F. Allen. A plan recognition model for subdialogues in conversations. Cognitive Science, 11(2):163-200, 1987.

[Lyon, 1974] Gordon Lyon. Syntax-directed least errors analysis for context-free languages. Communcations of the $A C M, 17(1): 3-14,1974$.

[Mckeown, 1985] K.R. McKeown. Text Generation. Cambridge University Press, New York, 1985.
[Morik, 1989] K. Morik. User models and conversationa! settings: Modeling the user's wants. In A. Kobsa and W. Wahlster, editors, User Models in Dialog Systems pages 364-385. Springer-Verlag, New York, 1989.

[Mudler and Paulus, 1988] J. Mudler and E. Paulus Expectation-based speech recognition. In H. Niemann M. Lang, and G. Sagerer, editors, Recent Advances it Speech Understanding and Dialog Systems, pages 473477. Springer-Verlag, New York, 1988.

[Novick, 1988] D.G. Novick. Control of Mixed-Initiativ Discourse Through Meta-Locutionary Acts: A Com putational Model. PhD thesis, University of Oregon 1988.

[Paris, 1988] C.L. Paris. Tailoring object descriptions tı a user's level of expertise. Computational Linguistics 14(3):64-78, 1988.

[Patten, 1988] T. Patten. Systemic Text Generation a Problem Solving. Cambridge University Press, Nev York, 1988.

[Polanyi and Scha, 1983] L. Polanyi and R. Scha. Oı the recursive structure of discourse. In $\mathrm{K}$. Ehlich an H. van Riemsdijk, editors, Connectedness in Sentence Discourse and Text, pages 141-178. Tilburg Univer sity, 1983.

[Pollack, 1986] M.E. Pollack. A model of plan inferenc that distinguishes between the beliefs of actors an observers. In Proceedings of the 24th Annual Meet ing of the Association for Computational Linguistic: pages 207-214, 1986.

[Reichman, 1985] R. Reichman. Getting Computers $t$ Talk Like You and Me. MIT Press, Cambridge, Mass. 1985.

[Smith, 1991] R.W. Smith. A Computational Model c Expectation-Driven Mixed-Initiative Dialog Process ing. PhD thesis, Duke University, 1991.

[Walker and Whittaker, 1990] M. Walker and S. Whil taker. Mixed initiative in dialogue: An investigatio into discourse segmentation. In Proceedings of the $28 t$ Annual Meeting of the Association for Computation. Linguistics, pages 70-78, 1990.

[Walker, 1978] D.E. Walker, editor. Understanding $S p c$ ken Language. North-Holland, New York, 1978.

[Whittaker and Stenton, 1988] S. Whittaker and P. Stenton. Cues and control in expert-client dic logues. In Proceedings of the 26th Annual Meeting । the Association for Computational Linguistics, page 123-130, 1988.

[Young and Proctor, 1989] S.J. Young and C.E. Pror tor. The design and implementation of dialogue cor trol in voice operated database inquiry systems. Con puter Speech and Language, 3:329-353, 1989.

[Young et al., 1989] S.R. Young, A.G. Hauptman] W.H. Ward, E.T. Smith, and P. Werner. High lev knowledge sources in usable speech recognition sy: tems. Communications of the $A C M$, pages 183-19. August 1989. 